\title{
USO DE DIFERENTES TÉCNICAS DE MEDIDA DA CONCENTRAÇÃO CELULAR DE Saccharomyces cerevisiae EM MEIO FERMENTATIVO
}

\author{
I. I. K. VELOSO ${ }^{1}$, N. S. VARGAS ${ }^{2}$, A. C. BADINO JUNIOR ${ }^{1,2}$, e A. J. G. CRUZ ${ }^{1,2}$, R. G. \\ SILVA ${ }^{1}$, \\ ${ }^{1}$ Universidade Federal de São Carlos, Departamento de Engenharia Química \\ ${ }^{2}$ Universidade Federal de São Carlos, Programa de Pós-Graduação em Engenharia Química \\ E-mail para contato: ivanveloso.eq@gmail.com
}

\begin{abstract}
RESUMO - O presente trabalho apresenta um estudo comparativo de diferentes métodos de medida da concentração celular durante a fermentação alcoólica. Foi utilizada nos cultivos a levedura Saccharomyces cerevisiae industrial liofilizada (fermento biológico instantâneo Y-904) a $34^{\circ} \mathrm{C}$. Para isso foram realizadas fermentações com concentração inicial de células de $15 \mathrm{~g} / \mathrm{L}$. Amostras foram retiradas a cada hora e submetidas a cinco métodos para obtenção da concentração celular: massa seca, densidade óptica e turbidímetro, cujos resultados são obtidos em g/L e Câmara de Neubauer e Moxi-Z cujos resultados são obtidos em $\mathrm{n}^{\circ}$ células/mL. As técnicas cujos resultados são obtidos em $\mathrm{g} / \mathrm{L}$ foram comparados ao método da massa seca. Os resultados do turbidímetro apresentaram a tendência esperada, porém não foram muito precisos devido à instabilidade durante as medidas. Os resultados obtidos por densidade óptica ficaram próximos aos obtidos pelo método da massa seca. Os resultados obtidos em $\mathrm{n}^{\circ}$ células $/ \mathrm{mL}$ utilizando o contador automatizado Moxi-Z foram comparados aos resultados obtidos utilizando-se a contagem em câmara de Neubauer. Após as adaptações de cada método de contagem celular obteve-se resultados com pequeno desvio padrão.
\end{abstract}

\section{INTRODUÇÃO}

Com o objetivo de diminuir a dependência das importações de petróleo e reduzir os efeitos da primeira crise do petróleo que afetou o Brasil em 1973, o governo federal incentivou o aumento da produção de etanol implantando o programa Proálcool em 1975. Depois de alguns altos e baixos, o programa voltou a ganhar força em 2003 principalmente devido à conscientização ambiental promovida pelo protocolo de Kyoto (1997), ao elevado preço internacional do petróleo e à introdução de veículos flex fuel no mercado brasileiro (Michellon et al., 2008). Com esse incentivo e com o clima tropical favorável ao plantio, o país se destaca na produção de biocombustíveis provenientes da cana-de-açúcar (Kohlhepp, 2010).

A produção mundial de etanol foi de 98 bilhões de litros em 2015 (UNICA, 2016). A maior parte desse montante foi produzido por fermentação utilizando a levedura Saccharomyces cerevisiae (Oliveira et al., 2014). Sabendo disso, é de extrema importância o 
estudo cinético de processos fermentativos utilizando essa linhagem de levedura para o aumento da produção de etanol no cenário atual. Para a análise cinética da fermentação é necessário acompanhar uma ou mais variáveis de processo (concentrações de biomassa, substrato ou produto de interesse).

A medida da concentração celular durante a fermentação permite analisar o crescimento das leveduras e consequentemente a produção de etanol em diferentes condições de processo, como $\mathrm{pH}$, temperatura, meio fermentativo, etc. Vale ressaltar ainda que a medida da concentração celular ao longo do cultivo é importante para monitorar o andamento da fermentação e identificar o seu término, quando a concentração celular apresenta valores máximos ou decai devido à morte celular (Hiss, 2001). Neste trabalho são comparadas diferentes técnicas de medidas para acompanhamento do crescimento das leveduras ao longo da fermentação alcoólica. Entre os métodos estão a massa seca, densidade óptica, turbidimetria e a contagem de números de células utilizando a câmara de Neubauer e um contador automatizado.

\section{MATERIAIS E MÉTODOS}

\subsection{Preparo do Inóculo}

O meio de cultivo em todas as fermentações foi composto por: sacarose (180 g/L), $\mathrm{KH}_{2} \mathrm{PO}_{4}(5,6 \mathrm{~g} / \mathrm{L}), \mathrm{MgSO}_{4} .7 \mathrm{H}_{2} \mathrm{O}(1,4 \mathrm{~g} / \mathrm{L})$, extrato de levedura $(6,8 \mathrm{~g} / \mathrm{L})$, ureia $(5,3 \mathrm{~g} / \mathrm{L})$ e quantidade suficiente de água destilada para completar $1 \mathrm{~L}$. O pH foi ajustado para 4,5 com $\mathrm{HCl}$ 1,0 M. Foi utilizada a levedura Saccharomyces cerevisiae liofilizada (fermento biológico instantâneo Y-904) com concentração inicial de células igual a 15 g/L.

\subsection{Fermentação Alcoólica}

Inicialmente foram realizadas fermentações de 5 horas em Erlenmeyers de $250 \mathrm{~mL}$ contendo $100 \mathrm{~mL}$ de meio de cultura, mantidos em incubadora shaker a $34^{\circ} \mathrm{C}$ e $200 \mathrm{rpm}$. Experimentos posteriores foram realizados em reator encamisado de $500 \mathrm{~mL}$ contendo 300 $\mathrm{mL}$ de meio de cultura, com tempo de cultivo de 9 horas a $34^{\circ} \mathrm{C}$ e agitação de $200 \mathrm{rpm}$ utilizando agitador e barra magnética. Por fim foram realizadas fermentações em biorreator de 5 L (BioFlo ${ }^{\circledR} 310$ ) contendo 4 L de meio de cultura, com duração de 9 horas, temperatura de $34^{\circ} \mathrm{C}$ e agitação de $250 \mathrm{rpm}$ pelo sistema motor-impelidor do próprio equipamento.

\subsection{Metodologia Analítica}

Amostras retiradas a cada hora da fermentação foram submetidas a cinco métodos de obtenção da concentração celular. Os métodos da massa seca, densidade óptica e turbidímetro fornecem resultados da concentração celular em $\mathrm{g} / \mathrm{L}$, enquanto os métodos utilizando a Câmara de Neubauer e o Contador Automatizado Moxi-Z fornecem resultados em $\mathrm{n}^{\circ}$ células/mL.

Massa seca (gravimétrico): Para obtenção da concentração celular pelo método da massa seca retirou-se uma alíquota de $2 \mathrm{~mL}$ do caldo fermentativo que foi centrifugada, seca e pesada determinando-se assim a massa de leveduras presente em $2 \mathrm{~mL}$ de amostra. Densidade óptica: $\mathrm{O}$ método da densidade óptica foi realizado de duas formas, método direto sem 
lavagem das células (D.O direto) e método com lavagem das células (D.O com amostras lavadas). Para os dois métodos o comprimento de onda emitida pelo espectrofotômetro foi ajustado para $600 \mathrm{~nm}$. Para a leitura de absorbância em espectrofotômetro pelo método da D.O direto a alíquota retirada da fermentação foi diluída 60 vezes com água destilada em balão volumétrico. As amostras submetidas ao método da D.O com amostras lavadas passaram pelos processos de centrifugação e lavagem das células antes de serem diluídas com água destilada no balão volumétrico para leitura no espectrofotômetro. Para converter a absorbância lida em concentração em g/L, foi necessária a obtenção das curvas de calibração para cada método. Turbidimentria: O turbidímetro da marca Mettler Toledo ${ }^{\circledR}$ foi calibrado antes do uso, inserindo-se o sensor do equipamento em quatro soluções de diferentes concentrações de $\mathrm{NaCl} 0,9 \mathrm{~m} / \mathrm{v}$ e leveduras. A concentração de cada solução foi determinada pelo método da D.O com amostras lavadas. Para comparar com os resultados da massa seca, a concentração celular fornecida pelo turbidímetro foi observada a cada meia hora, anotando-se 30 valores a cada medida, a fim de se observar a tendência de crescimento celular.

Câmara de Neubauer: Para a determinação do número de células utilizando a câmara de Neubauer, retirou-se uma alíquota de $30 \mu \mathrm{L}$ do caldo fermentativo que foi diluída em EDTA 0,1 M, agitada e colocada sobre a câmara contendo a lamínula. Com a câmara posicionada no microscópio óptico fotografou-se cinco quadrados médios da câmara, para estimar a quantidade total de células presentes na área de contagem, utilizando o software S-Viewer. A área de contagem de células é igual a $1 \mathrm{~mm}^{2}$. Quando colocada a lamínula sobre a câmara obtém-se uma distância de $0,1 \mathrm{~mm}$. Logo o volume de amostra em análise é de $0,1 \mathrm{~mm}^{3}$ (0,0001 mL). O número de células foi obtido pela Equação 1 .

$$
\mathrm{C}\left(\mathrm{n}^{\circ} \mathrm{cel} / \mathrm{mL}\right)=\frac{\left(\mathrm{n}^{\circ} \text { células contadas }\right) \cdot 25}{\mathrm{n}^{\circ} \text { quadrados médios contados }} \cdot \mathrm{F} \cdot 10^{4}
$$

Onde $10^{4}$ é o fator de conversão de unidades de $\mathrm{mm}^{3}$ para $\mathrm{mL}$ e $\mathrm{F}$ é o fator de diluição.

Moxi-Z: A concentração celular fornecida pelo equipamento Moxi-Z foi obtida diluindo-se 400 vezes uma alíquota de $30 \mu \mathrm{L}$ do caldo fermentativo em solução tampão fosfato salina (PBS). A solução diluída e agitada foi inserida no cassete do equipamento, fornecendo a concentração celular.

\section{RESULTADOS E DISCUSSÃO}

\subsection{D.O Direto, D.O com Amostras Lavadas e Massa Seca}

A Figura 1 mostra os resultados da concentração celular de um cultivo em biorreator BioFlo $^{\circledR}$ em que são comparados os resultados obtidos pelos métodos da D.O direto, D.O com amostras lavadas e massa seca 
Figura 1 - Resultados da concentração celular em g/L obtidos pelos dois métodos de D.O e pela massa seca.

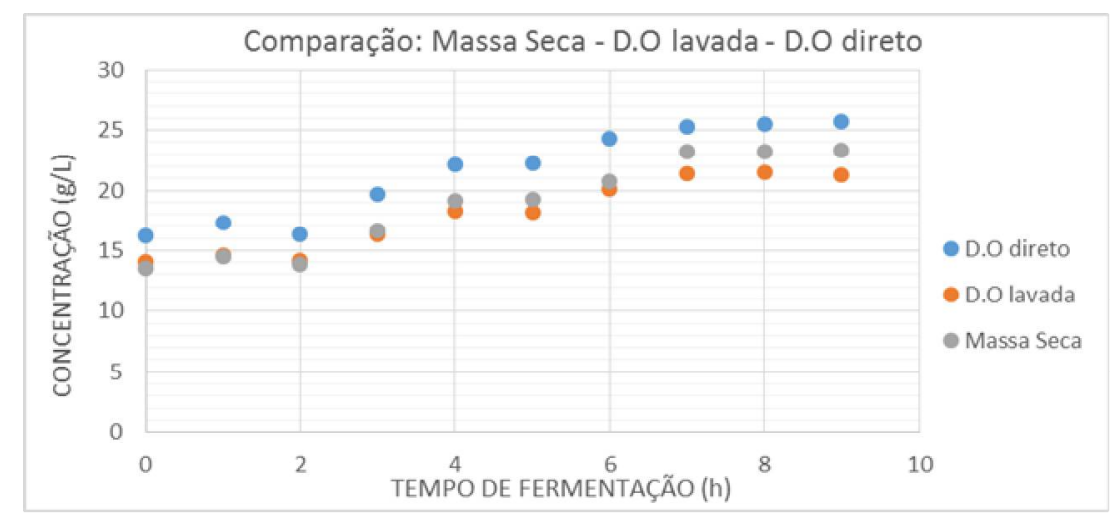

Pode-se observar que a concentração celular em g/L obtida pelo método da D.O com amostras lavadas se aproxima mais da concentração celular obtida pela massa seca. A discrepância entre os resultados obtidos pelo método da D.O direto é decorrente principalmente da presença de bolhas de $\mathrm{CO}_{2}$ na amostra. Quando a amostra é colocada no espectrofotômetro as bolhas interferem na leitura da absorbância, desviando o feixe de luz emitido pelo aparelho. Comparando o método da D.O com amostras lavadas e a massa seca não se observa esse descolamento dos dados pois as bolhas de $\mathrm{CO}_{2}$ foram eliminadas no processo de centrifugação e lavagem das células. Porém observa-se uma diferença entre os resultados das duas medidas a partir da sexta hora de fermentação. Provavelmente isso ocorra devido ao fato de que a absorbância lida no espectrofotômetro, a partir da sexta hora (altas concentrações), apresenta valor próximo ou superior a 0,900, fora da validade da Lei de Lambert-Beer (desvio da linearidade por altas concentrações).

\subsection{Turbidímetro e Massa Seca}

Os resultados obtidos utilizando o turbidímetro durante uma fermentação em reator encamisado de 500 mL são mostrados na Figura 2.

Figura 2 - Resultados da concentração celular em g/L obtidos pelo turbidímetro e pela massa seca na fermentação em reator encamisado de $500 \mathrm{~mL}$

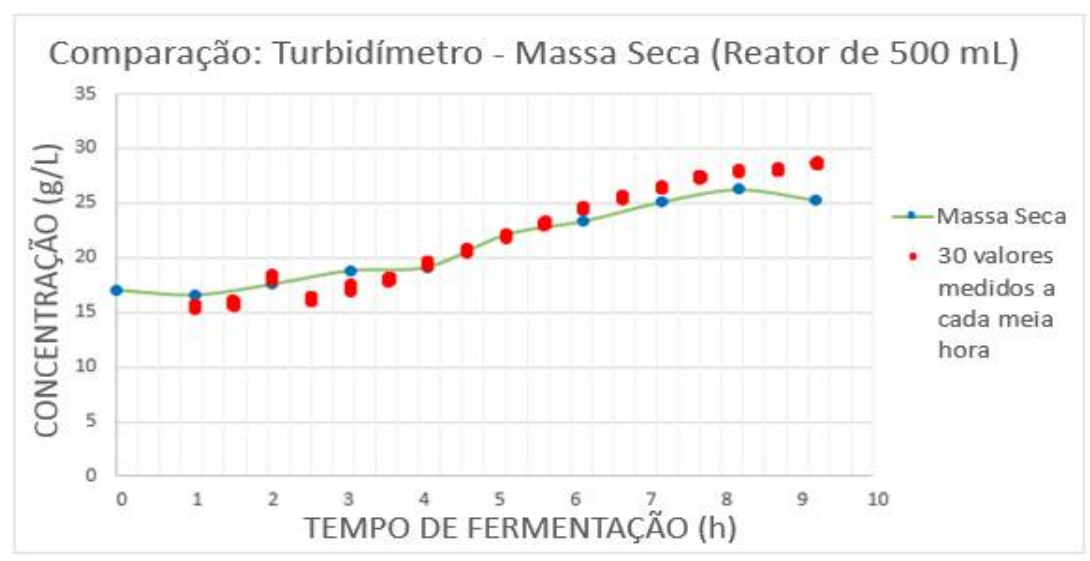


A concentração celular fornecida pelo equipamento em tempo real não apresentou valores estáveis dificultando a leitura de um único valor preciso. As oscilações são decorrentes principalmente pela presença de $\mathrm{CO}_{2}$ no processo. Entretanto pôde-se observar que os resultados obtidos pelo turbidímetro apresentaram a mesma tendência observada pelo método gravimétrico.

A partir da sexta hora observa-se um deslocamento em relação à massa seca que acredita-se ter ocorrido apelo fato da calibração do turbidímetro depender diretamente da leitura no espectrofotômetro. Dessa forma, como discutido anteriormente, as leituras de D.O para altas concentrações fogem da linearidade da Lei de Lambert-Beer e esse desvio foi propagado para o turbidímetro.

\subsection{Câmara de Neubauer e Moxi-Z}

Os resultados das concentrações celulares obtidas pelo equipamento Moxi-Z e pela contagem na câmara de Neubauer são mostrados na Figura 3.

Figura 3 - Resultados em no células/mL obtidos pela câmara de Neubauer e Moxi-Z.

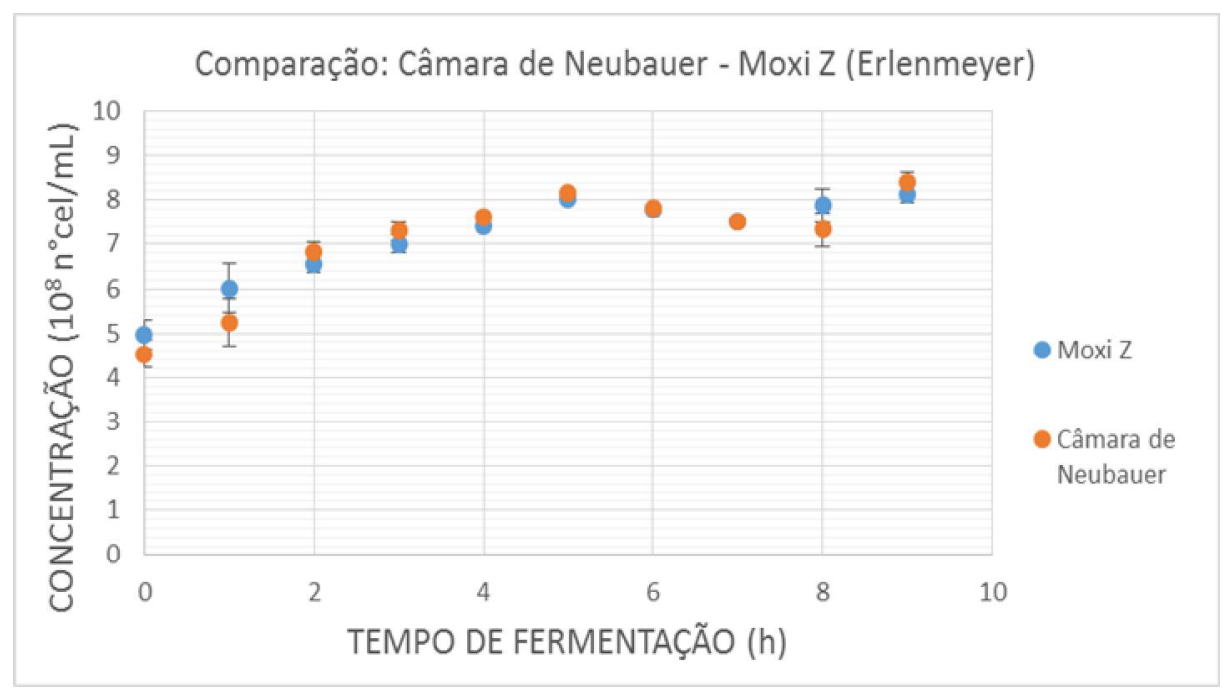

Comparando-se os resultados obtidos pela câmara de Neubauer e pelo Moxi-Z pode-se inferir que os dois métodos apresentaram resultados próximos. A discrepância observada entre alguns pontos podem ser atribuídas a erros de diluição e pipetagem, já que as alíquotas retiradas da fermentação possuíam volume de $30 \mu \mathrm{L}$. Quando se trata de diluição de amostras todo cuidado é pouco, pois por menor que seja o volume adicionado a mais ou a menos, promoverá resultados distantes da realidade. 


\section{CONCLUSÃO}

As medidas de concentração celular obtidas pelo método da densidade óptica, apresentaram resultados próximos aos obtidos pelo método da massa seca principalmente quando $\mathrm{o} \mathrm{CO}_{2}$ foi eliminado por lavagem das amostras e centrifugação. A vantagem do método da densidade óptica em relação ao método da massa seca é a rapidez na obtenção da concentração celular. Comparando-se os valores obtidos com o turbidímetro e a massa seca, observa-se o mesmo comportamento do crescimento celular ao longo da fermentação. Apesar das oscilações nas medidas, o turbidímetro apresentou resultados que descreveram em tempo real o crescimento celular ao longo do cultivo, sendo essa uma vantagem em relação ao método gravimétrico. Moxi-Z apresentou resultados muito próximos aos obtidos pela contagem em Câmara de Neubauer. A vantagem do Moxi-Z encontra-se na rapidez e facilidade de obtenção de resultados, porém o alto custo dos cassetes pode dificultar o uso rotineiro do método em laboratório.

\section{AGRADECIMENTOS}

Os autores agradecem ao CNPQ, CAPES e FAPESP pelo apoio financeiro.

\section{REFERENCIAS}

CARVALHAL, M. L. C. Técnicas Básicas em Microbiologia. In: BORZANI, W.; SCHMIDELL, W.; LIMA, U. A.; AQUARONE, E. Biotecnologia Industrial Fundamentos. v. 1, cap. 2, p. 33-62, 2001.

HISS, H. Cinética de Processos Fermentativos. In: SCHMIDELL, W.; LIMA, U. A.; AQUARONE, E.; BORZANI, W. Biotecnologia Industrial - Engenharia Bioquímica. v. 2, cap. 6, p. 93-122, 2001.

KOHLHEPP, G. Análise da situação da produção de etanol e biodiesel no Brasil. Estudos Avançados. v. 24, n 68, p. 223-253, 2010.

MICHELlON, E.; SANTOS, A. A. L.; RODRIGUES, J. R. A. Breve descrição do Proálcool e perspectivas futuras para o etanol produzido no Brasil. XLVI Congresso da Sociedade Brasileira de Economia, Administração e Sociologia Rural. p. 16, 2008.

OLIVEIRA, J. H. S.; SILVA, M. C. S.; SANTOS, M. S. R.; ALMEIDA, R. M. R. G. Cinética do crescimento de leveduras em meio sintético, na presença de inibidores, usando diferentes concentrações de fermento. XX Congresso Brasileiro de Engenharia Química.p. 8, 2014.

UNICA - União as Indústria de Cana-de-Açúcar. Portal UNICA 〈http://www.unica.com.br $\rangle$. Acessado em 25/12/2016. 